\title{
Conceptos Fundamentales Y Sus Propiedades Clave En Probabilidad - Cómo Identificarlos Y Proporcionar Intuiciones Que Los Sostengan
}

\author{
Fundamental Concepts And Their Key Properties In Probability \\ - How To Identify Them And Provide Intuitions That Support Them
}

\author{
Conceitos Fundamentais E A Sua Propriedades-Chave Em Probabilidade \\ - Como Identificá-Los E Fornecer Intuição Que Os Apoia
}

Manfred Borovenik ${ }^{*}$

Alpen-Adria-Universität Klagenfurt - AAU

\begin{abstract}
Resumen
La probabilidad está apoyada en conceptos teóricos, que están lejos de ser intuitivos. El primer paso es identificar los conceptos clave; el segundo es aclarar estos conceptos no sólo mediante el uso de herramientas matemáticas, ilustrando su valor en contexto o sus propiedades específicas. Nos centramos en las facetas de la probabilidad que permiten vincular sus diversas interpretaciones, o que vinculan la probabilidad a la inferencia estadística. Replanteamos problemas fundamentales de probabilidad, de los que surgen de la especificidad del concepto y de la finalidad para la que ha sido "diseñado". Esta especificidad requiere meta estrategias que van mucho más allá de la instrucción de los detalles matemáticos. En este trabajo, nos centramos en los aspectos teóricos de las ideas clave de la probabilidad y en cómo proporcionar intuiciones e imágenes que sean sostenibles.
\end{abstract}

Palabras clave: Interacción de intuiciones de Fischbein, Propósito de los conceptos, Estrategias arquetípicas, Pensamiento probabilístico, Teoremas centrales, Animaciones dinámicas, Alfabetización en probabilidad, Alfabetización en riesgo, Incertidumbre.

\begin{abstract}
Probability is highlighted by theoretical concepts, which are far from being intuitive. The first step is to identify key concepts; the second is to clarify these concepts not only using mathematical tools by illustrating either their value in context or their specific properties. We focus on facets of probability that allow for linking its various interpretations, or that link probability to statistical inference. We restate fundamental problems with probability that arise from the specificity of the concept and the purpose for which it has been "designed". This specificity requires meta strategies that go far beyond the instruction of the mathematical details. In this paper, we focus on theoretical aspects of key ideas of probability and how to provide intuitions and images that are sustainable.
\end{abstract}

Keywords: Fischbein's interplay of intuitions, Purpose of concepts, Archetypal strategies, Probabilistic thinking, Central theorems, Dynamic animations, Probability literacy, Risk, Uncertainty.

\footnotetext{
* Doctor in Statistics and its Didactics from the University of Klagenfurt (AAU). Professor at the Department of Statistics, Alpen-Adria Universität Klagenfurt (AAU), Klagenfurt, Austria. E-mail: manfred.borovcnik@aau.at
} 


\section{Resumo}

A probabilidade destaca-se por conceitos teóricos, que são distantes de ser intuitivos. O primeiro passo deve identificar conceitos-chave; o segundo deve clarificar estes conceitos não só utilização de instrumentos matemáticos ilustrando o seu valor no contexto ou as suas propriedades específicas. Concentramo-nos em facetas da probabilidade que levam em conta a vinculação das suas várias interpretações ou aquela probabilidade de conexão à inferência estatística. Declaramos novamente problemas fundamentais com a probabilidade que resultam da especificidade do conceito e o objetivo com o qual "se projetou". Esta especificidade necessita estratégias de Meta que vão longe além da instrução dos detalhes matemáticos. Neste artigo, concentramo-nos em aspectos teóricos de ideias-chave da probabilidade e como fornecer intuição e imagens que são sustentáveis.

Palavras-chave: A interação de Fischbein de intuição, Objetivo de conceitos, Estratégias arquetípicas, Pensamento probabilístico, Teoremas centrais, Animações dinâmicas, Alfabetização de probabilidade, Alfabetização dos riscos, Incerteza.

\section{Introducción}

Las dificultades con la enseñanza de la probabilidad están bien investigadas y descritas. Parece ser que mediante las innovaciones educativas de las dos últimas décadas cuando la educación estadística como comunidad y disciplina está creciendo enormemente y ganando influencia - la situación apenas ha mejorado. Sin embargo observamos un enfoque sesgado de las intervenciones de investigación y enseñanza basado en la idea de frecuencias relativas, que, de alguna manera, se podría interpretar como un intento de reducir el concepto de probabilidad al concepto de frecuencia relativa. Por supuesto, centrarse en la simplificación de los conceptos - como se hace con las frecuencias relativas - es una forma de mejorar la comprensión por parte de los alumnos. Sin embargo, algo debería llevar más allá de tales simplificaciones y puede ser que tengamos que aclarar los conceptos fundamentales de la teoría de la probabilidad y los problemas paradigmáticos en los que podemos trabajar y proporcionar soluciones parciales mediante conceptos probabilísticos.

Empleamos una forma hermenéutica de argumentar, relacionando las ideas fundamentales de la probabilidad con su trasfondo matemático y filosófico, para resaltar las ideas clave de la probabilidad. El objetivo es desarrollar un catálogo mínimo de ideas fundamentales que caractericen el concepto de probabilidad y revelar el propósito de los conceptos relacionados, siempre utilizando analogías y contextos para que puedan emerger percepciones estables de la probabilidad.

En la segunda sección, describimos enfoques didácticos para aclarar los conceptos clave, hacemos referencia a los paradigmas de la ciencia y estructuramos formas de abordar situaciones probabilísticas. Las interrelaciones entre los conceptos de una teoría, que 
normalmente se aclaran dentro de las matemáticas en un entorno axiomático, describen indirectamente el significado de los conceptos y los métodos que se construyen sobre estos conceptos. Para enseñar un concepto a alguien que aún no está familiarizado con él, es necesario simplificar sus propiedades matemáticas, y centrarse en imágenes clave o contextos paradigmáticos en los que se puedan utilizar los conceptos. Este procedimiento puede enriquecer - en un bucle de retroalimentación - también la teoría misma. La integración en los paradigmas de la ciencia permite reconocer el propósito y la relevancia más amplia de los conceptos y proporcionar un marco para la teoría, lo que también puede mejorar la comprensión de los alumnos del significado de los conceptos.

En la tercera sección, ampliamos las deliberaciones a las conexiones de la probabilidad y las situaciones bajo incertidumbre con la psicología. En otras ramas de las matemáticas, la psicología no tiene tanto espacio - aparte de los problemas de aprendizaje y cómo tratarlos. La situación es diferente en cuanto a la probabilidad y la estadística, en las que los conceptos se han desarrollado en una doble conexión con el riesgo, que está cerca del miedo y la expectativa, y con cuestiones de destino y adivinación. Ilustramos las relaciones entre probabilidad y psicología mediante un catálogo de estrategias que pueden describir el pensamiento probabilístico. También entramos en más detalles sobre las formas arquetípicas de pensar y las estrategias relacionadas (Batanero \& Borovcnik 2016) que pueden explicar los muchos conceptos erróneos sobre la probabilidad que permanecen incluso después de la enseñanza.

En la cuarta sección, explicamos con más detalle nuestro enfoque actual. Argumentamos que la probabilidad es un concepto teórico, que carece de toda posibilidad de materializarse sin perder su significado. Por lo tanto, retomamos la idea de una interacción dinámica entre las intuiciones y los conceptos matemáticos (véase Fischbein 1975, o Borovcnik 1992). Presentamos varias razones por las cuales las intuiciones incorrectas deben ser corregidas: primera los conceptos erróneos con probabilidad, que son bastante resistentes a la enseñanza; segunda, la falta de formas operacionales de adquisición de conceptos en caso de probabilidad; y tercera, la aparente dificultad para nombrar y describir las ideas fundamentales estocásticas. Aún más grave es la circunstancia de que las concepciones de la enseñanza a menudo están demasiado orientadas a las relaciones matemáticas de probabilidad, lo que no ayuda realmente a construir una comprensión sostenible de los conceptos probabilísticos. Se necesita algo más que las meras matemáticas para entenderlos.

Una vez que se reconoce la relevancia de las intuiciones correctas para entender el concepto de probabilidad, queda por abrir esta perspectiva mediante las intervenciones 
pedagógicas. Nos centramos en actividades de enseñanza que se apoyan en applets dinámicos, que extraen su valor a través de diversas formas de simulación, que exploran las consecuencias de los conceptos o métodos involucrados. Incrustamos el problema en un contexto, exponemos el propósito de los métodos y evitamos cualquier consideración obscura sobre límites de las frecuencias relativas. En lugar de investigar empíricamente los límites matemáticos, mostramos algunas características generales de los conceptos que parecen deseables y naturales en el contexto para que los métodos comiencen a tener SENTIDO.

Finalmente, sacamos algunas conclusiones y nos referimos a los applets dinámicos, que pueden apoyar intervenciones didácticas adecuadas para establecer percepciones sostenidas por parte de los alumnos, revelando el núcleo de los conceptos fundamentales de probabilidad. Este artículo forma parte de una trilogía de artículos sobre el complejo tema del valor de los conceptos fundamentales y cómo proporcionar intervenciones didácticas que apoyen la comprensión del concepto de probabilidad.

El nivel de las consideraciones está definitivamente dirigido a los dos últimos años antes del examen final al final del Gimnasio. Las ideas han sido probadas con profesores, estudiantes de magisterio, y con estudiantes de estudios no matemáticos (principalmente negocios y administración que son bastante débiles en matemáticas). Los ejemplos a los que se hace referencia son comparables a los de Batanero y Borovcnik (2016), Estadísticas y probabilidad en la escuela secundaria. De hecho, las consideraciones de este documento conforman la filosofía de fondo y explicitan las ideas que guiaron los ejemplos de Batanero y Borovcnik (2016). Las consideraciones apuntan a construir el meta conocimiento para entender los conceptos matemáticos, a veces difíciles, de probabilidad, que son esenciales para una comprensión más completa de la probabilidad.

\section{La Perspectiva De La Didáctica Y Los Paradigmas De La Ciencia}

Una teoría en general es un conjunto coherente de afirmaciones sobre un tema que permite describir y modelar una parte de la realidad con el objetivo de explicar el statu quo en la realidad, predecir el curso futuro del desarrollo o detectar "causas" (razones) del estado o desarrollo actual. Las interrelaciones entre los conceptos de una teoría - que normalmente se aclaran dentro de las matemáticas en un entorno axiomático - describen indirectamente el significado de los conceptos y los métodos que se construyen sobre estos conceptos. Es útil añadir la perspectiva didáctica y el punto de vista de los paradigmas de la ciencia para arrojar 
más luz sobre los conceptos y facilitar la comprensión. Para enseñar un concepto a alguien que aún no está familiarizado con él, es necesario simplificar las propiedades matemáticas, o centrarse en imágenes clave o contextos paradigmáticos en los que los conceptos parecen más familiares. Este procedimiento puede enriquecer - en un bucle trasero - también la teoría misma. La integración en los paradigmas de la ciencia proporciona un marco para la teoría, que permite reconocer su propósito y relevancia más amplios, así como puntos de vista y teorías alternativos que compiten con el enfoque actual, lo que también puede mejorar la comprensión de los alumnos en cuanto al significado de los conceptos.

\subsection{Enfoques Didácticos Para Aclarar Los Conceptos Clave De Los Estocásticos}

La aceptación del azar como parte del mundo real, es una característica de la vida moderna, ya que hoy en día no se toma en forma fatalista, que se debe a que la vida es fundamentalmente incierta. Un aspecto de la toma de control sobre la incertidumbre está arraigado con una visión de probabilidad y modelos relacionados. Hubo épocas y culturas que aceptaron la incertidumbre o la trataron de manera diferente. Las preguntas introductorias que guían a Bennett (1999, p. 9) a través de su análisis del azard muestran los aspectos pluralistas de la probabilidad:

[...] examinar el azar y varias otras nociones que fueron críticas para el desarrollo histórico del pensamiento probabilístico - y que también juegan un papel importante en la comprensión (o malentendido) de las leyes del azar por parte de cualquier individuo. Investigamos una serie de ideas:

¿Por qué, desde la antigüedad hasta hoy, se ha recurrido al azar en la toma de decisiones?

¿Es una decisión tomada al azar una decisión justa?

¿Qué papel ha jugado el juego en nuestra comprensión del azar?

¿Son probables los eventos extremadamente raros a largo plazo?

¿Por qué algunas sociedades e individuos rechazan el azar?

¿Existe el verdadero azar?

¿Qué contribución han hecho los ordenadores al pensamiento probabilístico moderno?

¿Por qué incluso los expertos no están de acuerdo sobre los muchos significados de la aleatoriedad?

¿Por qué la probabilidad es contraintuitiva?

Batanero (2016) relaciona las siguientes facetas con el azard y su formalización:

Oportunidad y causalidad

Conceptos modernos del azar

Aleatoriedad como equiprobabilidad

Aleatoriedad como estabilidad de las frecuencias

Vistas subjetivas de la aleatoriedad

Axiomatización y vistas matemáticas formales 
De todos modos, la incertidumbre no se puede eliminar; sólo las consecuencias materiales de la incertidumbre se controlan de alguna manera.

La probabilidad es un concepto teórico, que dista mucho de ser intuitivo. Son bien conocidas las dificultades en el aprendizaje de la probabilidad y las abundantes concepciones erróneas relacionadas con la probabilidad, que son resistentes a los esfuerzos de enseñanza. Los conceptos matemáticos, que podrían aclarar las concepciones erróneas, son bastante complejos, por lo que a menudo se pierden el objetivo de superar los obstáculos conocidos de la probabilidad. En consecuencia, es necesario identificar los conceptos clave y aclararlos no sólo mediante el uso de herramientas matemáticas. Ilustramos los conceptos y su valor y propósito estableciendo contextos y analogías relevantes y desarrollando un acceso más directo a sus propiedades específicas a un nivel intuitivo. Para aclarar sus propiedades, también implementamos animaciones dinámicas para explorar la influencia de ciertos parámetros o datos en comparación con una situación inicial.

El carácter teórico de la probabilidad requiere meta-estrategias que vayan mucho más allá de los detalles matemáticos. Desarrollamos tareas adecuadas y animaciones interactivas para hacer frente a los obstáculos. La intención didáctica del presente documento es centrarse en conceptos fundamentales y describir sus propiedades mediante actividades que van más allá de las matemáticas. Utilizamos varias fuentes para ello:

- Nos concentramos en las intuiciones y en la interacción entre las intuiciones y los conceptos matemáticos en el sentido de Fischbein $(1975,1987)$, lo que permite un acceso directo a los conceptos que revelan las matemáticas. Borovenik (1992) demuestra lo útil que es desarrollar intuiciones secundarias basadas en los conceptos adquiridos, que pueden superar las intuiciones primarias, a menudo engañosas, sobre la probabilidad y al mismo tiempo permitir atajos intuitivos para comprender las propiedades clave de los modelos y conceptos estocásticos, así como los resultados, al menos de forma cualitativa ${ }^{1}$. Utilizamos analogías que tienen, además de un aspecto motivacional positivo, también una función de concretización, estructuración y asimilación (Simons 1984), lo que las hace útiles en la adquisición de conceptos. Esto se aplica especialmente a la probabilidad, ya que el concepto es genuinamente teórico (Steinbring 1991).

\footnotetext{
${ }^{1}$ En el sentido de reconocer una influencia de algún parámetro, no en el sentido de obtener resultados numéricos exactos.
} 
- Revelar el propósito del concepto puede mejorar las percepciones individuales sobre sus propiedades, que incluso pueden ser "esperadas". De este modo, volvemos a plantear los problemas fundamentales de probabilidad que surgen de la especificidad de este concepto y de la finalidad para la que ha sido "diseñado". Si abordamos el propósito de la probabilidad, también tenemos que reconstruir las estrechas interrelaciones entre probabilidad y riesgo y toma de decisiones, que históricamente fueron la fuerza motriz para desarrollar conceptos probabilísticos (Borovenik \& Kapadia 2018, Borovcnik 2015).

- Rastrear el surgimiento histórico de los conceptos, especialmente las estrechas relaciones entre probabilidad y riesgo, está abriendo la mente. La probabilidad y el riesgo pueden incluso considerarse conceptos gemelos (Borovcnik \& Kapadia 1991, 2018). La probabilidad se ha configurado para comparar riesgos y decidir para mejor de varias opciones. Podemos aprender de la historia; también podemos aprender de la historia de los enfoques didácticos. En cierto modo, la historia de la enseñanza refleja el surgimiento de los conceptos. No son sólo las modas las que influyen en la forma de enseñar y los enfoques didácticos (Jones, Langrall, \& Mooney 2007); estas formas expresan la creciente conciencia de otros factores de influencia en la construcción de la comprensión conceptual con los alumnos.

- Desarrollamos un catálogo de componentes mínimos de los conceptos estocásticos y sus propiedades. Existen algunos esfuerzos para identificar y describir la alfabetización probabilística, que también pueden interpretarse como conceptos mínimos que deberían incluirse en la enseñanza (e.g., Heitele, 1975, Batanero y Borovcnik, 2016), en forma intuitiva para que sean ampliamente accesibles y garanticen un efecto sostenible de la enseñanza de la probabilidad, de modo que los estudiantes puedan más tarde enfrentarse a situaciones de la vida real y aplicar la probabilidad y los conceptos relacionados para su comprensión de los problemas cotidianos. Además de la tentativa de Gal (2005), varias ideas del autor están basadas en consideraciones generales o ejemplos (Borovcnik 2006). Un aspecto de los modelos de probabilidad para resolver problemas del mundo real es que hay una idea clave detrás de cada una de las distribuciones de probabilidad, que es esencial para obtener resultados 
razonables de dichos modelos de probabilidad e interpretarlos de manera apropiada (Borovenik \& Kapadia 2011).

- Abogamos por la clarificación de las ideas a través de la discusión abierta y su puesta en práctica mediante applets dinámicos, que también pueden ir acompañados de analogías. Estas analogías tienen el potencial de revelar el propósito de los conceptos, de modo que sus propiedades subsiguientes sean aclaradas y las interrelaciones entre los conceptos sean más fáciles de entender. En particular, mostramos tareas adecuadas y animaciones interactivas, que están diseñadas para superar los obstáculos de aprendizaje y han sido revisadas y complementadas después de la evaluación de su uso intensivo en la enseñanza. El valor de la discusión en el aula, también en forma de una entrevista empírica e intervencionista no puede subestimarse.

\subsection{Paradigmas De La Ciencia}

Para identificar el carácter de los conceptos de una teoría, es útil tener una visión panorámica de cómo se utilizan estos conceptos y para qué propósito. ¿Cuáles son los paradigmas filosóficos de la ciencia que regulan el surgimiento de estos conceptos (ver Batanero, Chernoff, Engel, Lee, \& Sánchez, Batanero, Henry, \& Parzysz 2005, Hacking 1975, y van Plato 1994)? Para la probabilidad, son definitivamente las situaciones de lo incierto, ya sea en el futuro o en el pasado. Especialmente cuando se trata de tomar precauciones para protegerse contra impactos severos en el futuro, los modelos de probabilidad permiten - en uno de varios enfoques - analizar la situación y proporcionar decisiones.

La aleatoriedad pura puede definirse cómo la falta de control o de método para predecir el resultado futuro con certeza. Podemos ver la aleatoriedad (filosóficamente, el azar) como una idea incrustada en varios paradigmas que compiten con la forma de predecir el futuro y explicar el proceso de desarrollo de una variable de interés. Para simplificar, Borovcnik (2011, p. 74) ve tres paradigmas principales (figura 1; ver también Bennet, 1999 o Batanero, 2016).

Azar y adivinación: el azard es la expresión de la voluntad de Dios.

Azar y causalidad: el azard carece de causas o razones específicas, a diferencia de la causalidad, que incorpora condiciones específicas en un experimento.

Evolución aleatoria contra creacionismo: evolución sin razones específicas contrapuesto a la creación por un ser omnipotente o Dios.

Existen múltiples interrelaciones entre los paradigmas representados por las esquinas 
del triángulo didáctico de la figura 1.

"Si existe un Dios que todo lo sabe, surge la pregunta de si esto puede reconciliarse con el azar y el libre albedrío. Como el azar significa impredecibilidad, entra en conflicto con la omnisciencia. [...] El debate entre la teoría evolutiva de Darwin y el creacionismo es similar al debate de la causalidad-azar en la física. ¿La naturaleza es creada por un ser sobrenatural o ha evolucionado sólo por efectos aleatorios?"

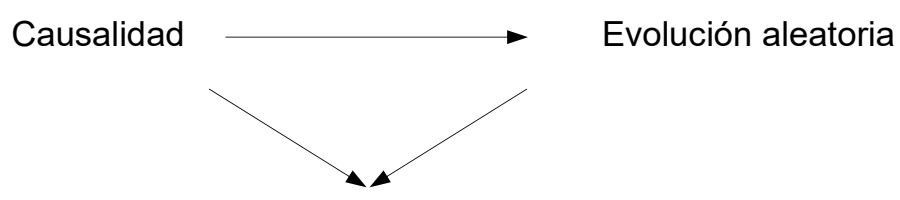

Adivinación

Figura 1: Triángulo didáctico que describe la interdependencia de los aspectos básicos de la probabilidad Fuente: Elaborado por el autor

La interconexión entre la causalidad y la aleatoriedad contrasta con los esfuerzos más recientes para eliminar la causalidad de la teoría de la física (Dürr, Goldstein, Tumulka, \& Zanghi 2004, o Styer 2000). El papel históricamente eminente de la esquina de adivinación se discute en David (1962) y es corroborado por Borovcnik y Kapadia (2014). La dualidad entre el libre albedrío y la evolución no ha estado en el centro de las consideraciones didácticas sobre la probabilidad, pero definitivamente tiene su efecto sobre la comprensión de la probabilidad, ya que también afecta el concepto de causalidad y en el fondo del azard, si se acepta la idea de un Dios que todo lo sabe.

Aceptar el demonio descrito por Laplace (1814) que lo sabía todo y aplica leyes causales para predecir el futuro nos dejaría atrás y nos dejaría usar la probabilidad como un sustituto de este conocimiento más amplio, ya que no conocemos los antecedentes. Sólo necesitaríamos probabilidad mientras no hayamos detectado las leyes causales detrás del fenómeno investigado.

La adivinación haría que la probabilidad fuera superflua, como si Dios decidiera que no corresponde a los seres humanos cuestionar e investigar sus decisiones. Este punto de vista podría explicar la conceptualización relativamente tardía de la probabilidad (véase Borovcnik \& Kapadia 2014). Por otra parte, el azar como un simple desarrollo sin ninguna razón más profunda impediría que una persona influyera - de cualquier manera, perceptible - en el curso de una acción para que uno pudiera interpretar el resultado final como una decisión de Dios; de hecho, esto se ha aplicado en los antiguos oráculos tales como Delfos. Históricamente, el hábito de externalizar una decisión crucial, como la elección de una persona, también se remonta al 
azar para "escuchar" la decisión de Dios (y evitar la responsabilidad de la decisión). Este aspecto de la probabilidad coincide con la idea de equidad e igualdad de oportunidades que han jugado un papel eminente en el surgimiento del concepto.

Otra conexión en el triángulo didáctico (figura 1) aparece entre la evolución aleatoria y el creacionismo (relacionado con la esquina de adivinación): La evolución de Darwin explica el curso del mundo mediante el azar (es decir, sin ninguna razón específica) ${ }^{2}$ mientras que el enfoque del creacionismo explica la existencia del mundo como creado por Dios. El debate entre la teoría de la evolución y el creacionismo reproduce en parte el debate de la causalidadaleatoriedad en la física. Esta extraña incrustación de probabilidad requiere intervenciones de enseñanza no convencionales, incluyendo una discusión sobre las formas arquetípicas de pensar.

\section{La Perspectiva De La Psicología}

En esta sección, desarrollamos un catálogo de estrategias para describir el pensamiento probabilístico que vincula los conceptos con la idea de riesgo. Entramos en más detalles sobre un tipo de estrategias, que Batanero y Borovcnik (2016) han denominado formas arquetípicas de pensar y sobre las estrategias relacionadas que parecen explicar por qué existen tantas concepciones erróneas sobre la probabilidad y por qué son tan robustas a pesar de las intervenciones de enseñanza. Concluimos esta sección con sugerencias preliminares para la enseñanza.

\subsection{Las Dobles Relaciones Entre La Probabilidad Y La Psicología}

Muchos problemas en la enseñanza de la probabilidad pueden estar relacionados con consideraciones emocionales y psicológicas. Así lo demuestran nuestras propias investigaciones, basadas en estudios históricos y entrevistas en profundidad (Borovcnik \& Bentz 1990 y 2003), las de Kahneman y Tversky - que se resumen en Kahneman, Slovic y Tversky (1982) - y muchas otras posteriores, como las incluidas en Chernoff y Sriraman (2014). Algunas de las razones por las que la enseñanza de la probabilidad es inevitablemente problemática se ponen de manifiesto en las fuentes históricas sobre la emergencia del concepto

\footnotetext{
${ }^{2}$ Un poco más preciso: La evolución de Darwin explica el curso del mundo mediante una componente aleatoria: unidad a las leyes de la herencia, competición entre sujetos y especies y sobrevivencia de los mejor adaptados.
} 
de probabilidad y sus diferentes facetas (Borovenik \& Kapadia 2018). La probabilidad ha surgido en una relación mutua con el riesgo, que siempre está ligado a los temores y expectativas sobre el resultado futuro.

Consistentemente, la alfabetización probabilística es descrita por Borovcnik y Kapadia mediante términos que tienen una amplia superposición con la psicología y el mundo de las intuiciones. ${ }^{3}$ Estos autores establecen dos niveles jerárquicos de categorías de pensamiento probabilístico (pp. 15):

\section{Categorías básicas del pensamiento probabilístico: intuiciones y estrategias}

1. La capacidad de discriminar entre aleatoriedad y causalidad;

2. La habilidad de equilibrar entre los elementos psicológicos y formales de la probabilidad;

3. La comprensión de que no existen criterios directos para el éxito en la probabilidad;

4. La comprensión de que los criterios para reflexionar sobre una situación aleatoria difieren de los que pueden aplicarse en la selección de una decisión.

\section{Categorías superiores intuiciones secundarias}

5. El conocimiento del carácter teórico de la probabilidad (combinando los aspectos SJT, $\mathrm{APT}$ y $\mathrm{FQT}^{4}$ ), e incluyendo el caso de probabilidades pequeñas;

6. La conciencia de la probabilidad condicional y su asimetría;

7. El desarrollo de conceptos que se construyen sobre evidencia probabilística (como la dependencia probabilística conceptualizada por el coeficiente de correlación).

Vale la pena señalar que Borovcnik y Kapadia (2018) formulan las intuiciones como competidoras, lo que significa que no está claro cuál de las intuiciones dominará el pensamiento. El azard y la causalidad (punto1) entran en conflicto con la adivinación (véase la figura 1); la capacidad de equilibrio entre los elementos psicológicos y los más formales (punto 2) aborda directamente el problema de qué tipo de pensamiento está más arraigado en los individuos y parece que existen en nosotros formas de pensar muy fuertes y robustas que dificultan la estabilidad del aprendizaje de la probabilidad. Cuando los criterios de éxito son discutibles (los epígrafes 3 y 4), falta una retroalimentación clara sobre el éxito y el fracaso de los conceptos aplicados, lo que puede obstaculizar el progreso conceptual y favorecer una visión personal de los problemas. Todas las intuiciones de orden superior ( $\left.\mathrm{n}^{\mathrm{o}} 5-7\right)$ se refieren a conceptos matemáticos y filosóficos sofisticados, por lo que no es de esperar que estén realmente maduros por el mero hecho de enseñarlos en su parte matemática.

\footnotetext{
${ }^{3}$ Ver también la sección 4 donde entramos en más detalles sobre el papel de las intuiciones en la enseñanza.

${ }^{4}$ Véase la sección 4.1 para el uso de esta terminología.
} 


\subsection{Formas Arquetípicas De Abordar Situaciones Probabilísticas}

A continuación, resumimos las ideas de Batanero y Borovcnik (2016) que se fundamentan en la investigación de Kahneman, Slovic y Tversky (1982), que resume trabajos realizados a partir de principios de la década de 1970 (entre otros, Tversky y Kahneman 1974, Kahneman y Tversky 1972, 1973 y 1979). Discernimos estrategias arquetípicas que son principalmente cognitivas y otras que son psicológicas. Por supuesto, hay interferencias mutuas y se despliegan múltiples estrategias al mismo tiempo. Lo que está causando más problemas para el aprendizaje y la planificación de las intervenciones de enseñanza es que estas estrategias se utilizan principalmente de forma inconsciente y cualquier intento de verbalizarlas es propenso al fracaso (véase también Borovcnik \& Bentz 1990, 2003).

\section{Estrategias arquetípicas de orientación cognitiva de abordar situaciones probabilísticas}

- Búsqueda de conexiones causales. Si uno encuentra una conexión causal entre los sucesos involucrados, las probabilidades que se deben calcular son mucho más claras o incluso superfluas. Las probabilidades explicadas causalmente están muy sobrevaloradas, mientras que no causales a menudo se ignoran.

- Búsqueda de patrones y similitudes. Esto incluye estrategias como la representatividad, por la cual un juicio de probabilidad es influenciado por una percepción de la similitud con el proceso de generación que está en segundo plano. Cada tipo de patrones puede ocurrir en secuencias aleatorias, de modo que ningún patrón puede indicar cualquier información (aparte de la situación en la que la suposición de aleatoriedad puede ser violada).

- La facilidad de acceso a la información, que resume las heurísticas de la disponibilidad y del anclaje, tienen una fuerte influencia en el juicio personal de las probabilidades. De acuerdo con la estrategia de disponibilidad, la probabilidad se aproxima intuitivamente a la facilidad de recordar casos relevantes. Sin embargo, no hay nada más poco fiable que la memoria personal. Según el fenómeno del anclaje, los juicios de probabilidad están muy influenciados por la información dada. Aquí, la información podría ser arbitrariamente puesta en una conexión oportuna con un incidente a juzgar.

La estrategia de búsqueda de patrones es errónea en caso de problemas estocásticos, donde las investigaciones revelan intuiciones incorrectas e inestables (ver Kahneman, Slovic, \& Tversky 1982, o Chernoff 2013). Todo es posible con el azar. Si se aplica la suposición básica 
de la aleatoriedad, entonces cualquier (extraño) patrón puede ocurrir sin ninguna ganancia en información. Por lo tanto, la búsqueda de patrones no ayuda; al menos no ayuda mientras el carácter aleatorio de la situación no esté seriamente en duda (Borovcnik \& Bentz 1990, 2003). Por lo tanto, los primeros análisis de probabilidad en la enseñanza deberían centrarse en los resúmenes estadísticos no en los patrones de cómo se generaron realmente los resultados.

\section{Estrategias arquetípicas de orientación psicológica}

- Búsqueda de la equidad o delegación de responsabilidades. Históricamente, se usó la aleatoriedad para externalizar las decisiones cruciales (Borovcnik \& Kapadia 2014). La preferencia personal en el Problema de las Tres Puertas o Monty Hall (por ejemplo, Borovcnik 2012) es quedarse con la primera elección de una de las tres puertas (una alberga el premio, las otras dos están "vacías"), aunque racionalmente sea mucho mejor cambiar la primera elección después de que una de las puertas restantes haya sido abierta y revelada como "vacía". Lo suficientemente extraño es que la gente se aferra emocionalmente a su primera elección, no siendo capaz de considerar argumentos racionales y defiende ferozmente (aunque sin argumento) su decisión (ver Borovcnik 2012).

- Usar la probabilidad para evitar la responsabilidad y obtener equidad: Si la elección entre varias opciones fuera equitativa, se podría adoptar un experimento aleatorio simétrico (como lanzar una moneda o un dado). La idea de equitatividad también constituye la base de la primera definición de probabilidad de Laplace (con la regla resultante de casos favorables divididos por casos posibles). Además de la equitatividad, la igualdad de probabilidades también se argumenta por la falta de razones específicas a favor de una de las posibilidades. La imparcialidad también puede dominar el sesgo de la equiprobabilidad, que es una tendencia a juzgar posibilidades como iguales, especialmente si hay dos opciones (el sesgo ha sido descrito por Lecoutre 1992; véanse también las entrevistas en Borovcnik \& Bentz 1990 y 2003).

- Reemplazar la información imitando a otras personas (el efecto de pastoreo). Un juicio personal (y una decisión basada en el) puede ser influenciada por lo que otros hacen. En retrospectiva, la información puede ser reinterpretada para encajar y justificar las acciones elegidas, lo que se denomina sesgo de confirmación: hay una fuerte tendencia de los seres humanos a justificar sus decisiones reinterpretando o incluso ignorando los hechos y acontecimientos 
posteriores para que su decisión siga pareciendo la mejor (para más detalles, véase Borovcnik \& Kapadia 2018).

\subsection{EI Valor Didáctico De Las Estrategias Arquetípicas}

Las formas arquetípicas de abordar los problemas de probabilidad no cambian con la educación. Hay muchos estudios que indican que incluso las personas bien educadas regresan a estas estrategias "primitivas" cuando están sobrecargadas cognitivamente. ${ }^{5}$ Para cualquier esfuerzo educativo en probabilidad (y estadística) es esencial considerar formas arquetípicas de pensar que han tenido éxito en otros campos, pero que son mucho menos prometedoras en situaciones estocásticas simplemente por su naturaleza, que se caracteriza genuinamente por un elemento de imprevisibilidad. Las estrategias que se han descrito en la última sección deben ser consideradas cuidadosamente en la enseñanza. Por supuesto, las suposiciones de fondo de una situación pueden ser simuladas y al ser confrontados con la baja tasa de éxito de sus estrategias en problemas aleatorios artificiales, los estudiantes pueden reconsiderar su enfoque. Sin embargo, lo que es más importante, una discusión abierta en el aula sobre los méritos relativos de tales estrategias podría ser mucho más útil para convencer a los alumnos de que sus estrategias son con bastante frecuencia inapropiadas en situaciones estocásticas.

Hay dos intervenciones de enseñanza que podrían ayudar a superar las estrategias arquetípicas: la simulación y la discusión de las ideas relevantes en la forma de una entrevista dirigida (no una forma neutra, sino una forma orientada de entrevista empírica, tal como se utilizó como método de investigación en Borovcnik \& Bentz 1990 y 2003).

- Las suposiciones de fondo de una situación aleatoria pueden ser simuladas y por un bajo índice de éxito repetido de tales estrategias en problemas aleatorios artificiales, los estudiantes pueden reconsiderar su primer enfoque.

- Discutir ideas sobre los méritos relativos de tales estrategias podría convencer a los alumnos de que sus estrategias son inapropiadas en situaciones estocásticas.

Tales estrategias deben ser corregidas enseñando los conceptos formales de manera que los alumnos tengan un acceso intuitivo directo al nivel formal de los conceptos.

\footnotetext{
${ }^{5}$ Digamos, al menos por la presión del tiempo que impide un análisis completo; ver, por ejemplo, Borovcnik \& Bentz 1990 y 2003, Gigerenzer 2002, o Dubben \& Beck-Bornholdt 2010.
} 


\section{El Enfoque Actual}

Las intuiciones son variadas, difíciles de verbalizar y pueden ser confusas. Sin embargo, excluir el tratamiento de las intuiciones en la enseñanza y centrarse directamente en la parte matemática de los conceptos, podría tener un impacto severo en la comprensión, ya que las intuiciones siempre serían más fuertes que los conceptos matemáticos artificiales y dificultarían el pensamiento claro con las concepciones formadas por los conceptos matemáticos. En esta sección, abogamos por un papel clave de las intuiciones en la enseñanza. Nos referimos al marco de Fischbein (1975) y desarrollamos criterios para desarrollar material didáctico dinámico que abarque una interacción entre las intuiciones y los conceptos teóricos (Borovcnik 1992).

\subsection{Las Intuiciones, Las Ideas Fundamentales Y EI Pensamiento Estocástico}

La probabilidad es un concepto teórico. Esto implica que las interpretaciones individuales de la misma siempre pasan por alto el concepto completo e incluso pueden sesgar su comprensión. Esto implica también que el concepto carece de materializaciones o visualizaciones simples que ilustren el concepto y realcen su significado. Steinbring (1991) se ha referido en primer lugar al genuino carácter teórico de la probabilidad y ha argumentado que el concepto se desmoronaría si la enseñanza desarrollara sólo un aspecto de sus componentes complementarios.

Este carácter teórico se ve reforzado por las afirmaciones de Spiegelhalter (2014a, b) que no sólo destaca su complejidad, sino que también habla de un "concepto metafórico". Este punto de vista no sólo explica las dificultades relativas que los alumnos experimentan con la probabilidad y que el concepto - aunque básicamente es una noción matemática - es tan profundamente malinterpretado. Esto puede ser una señal de que los esfuerzos educativos no intervienen en el mundo de las intuiciones de los individuos como si los conceptos de probabilidad se adquirieran a un nivel avanzado con signos vacíos de significado. O bien, las materializaciones y visualizaciones implantadas para la enseñanza pasan por alto el punto de la probabilidad, ya que reflejan una versión sesgada del concepto.

Borovcnik (1992) es un intento de abordar el carácter teórico de la probabilidad desarrollando una interacción dinámica entre las intuiciones y los conceptos matemáticos utilizando ideas de Fischbein $(1975,1987)$. Según el enfoque de Fischbein, las intuiciones son 
la fuerza motriz en el desarrollo de conceptos teóricos; determinan la forma de los conceptos y los contextos en los que pueden ser útiles para resolver problemas. De esta manera, las intuiciones se convierten en la clave para entender la teoría, ya que la teoría puede ser considerada como un conjunto de intuiciones congeladas, despejadas por algún argumento lógico y aumentadas para completarlas. Lo que complica las cosas con la probabilidad es que existen intuiciones divergentes y competitivas y el hecho de que pueden ser apoyadas y justificadas por diferentes teorías matemáticas de la probabilidad.:

- FQT: Una teoría es la teoría axiomática habitual que se remonta a Kolmogorov (1933), que retoma y justifica la idea de frecuencias relativas idealizadas (y evita los problemas resultantes de la "definición" de probabilidad como límite de frecuencias relativas). Usamos el acrónimo FQT para referirnos a la visión frecuentista de la probabilidad que está relacionada con la teoría de la probabilidad de Kolmogorov. ${ }^{6}$

- SJT: Otro enfoque se basa en una derivación matemática de una medida de probabilidad a partir de un sistema de preferencias formulado axiomáticamente, que retoma la idea de decisiones racionales entre diferentes opciones inciertas (de Finetti 1937, 1974). Usamos las letras SJT para denotar la visión subjetivista (no para confundir con la subjetiva) de la probabilidad. Para el uso de esta terminología para diferenciar entre los puntos de vista modernos sobre la probabilidad, véase Borovcnik y Kapadia (2014).

- APT: Además de la visión FQT y SJT, existe otra visión sobre la probabilidad ligada a la equiprobabilidad según Laplace con la regla de los casos favorables dividida por los posibles casos para los cálculos de probabilidad. Esto - lo llamamos APT (a priori teoría de la probabilidad; a priori ya que es el único que proporciona directamente valores de probabilidad para los eventos que se investigan) - la visión de APT es didáctica e históricamente una visión

\footnotetext{
${ }^{6}$ Aunque Kolmogorov (1933) afirmó persistentemente que el concepto de probabilidad establecido por su sistema de axiomas está libre de cualquier interpretación, básicamente utilizó la interpretación de frecuencia para formular su teoría. También derivó la ley de los grandes números ya en su documento seminal (p. 57), aunque no la mencionó explícitamente como justificación de la interpretación frecuentista de la probabilidad. El éxito de su trabajo de 1933, sin embargo, debe ser visto desde la perspectiva de las necesidades de la física; había resuelto el problema de una base satisfactoria de la probabilidad, que es capaz de ser interpretada por un frecuentisto sentido y de sortear los obstáculos que von Mises (1919) había enfrentado con su definición axiomática directa de la probabilidad para colectivos y el límite de las frecuencias relativas (idealizadas). En la controversia sobre los fundamentos en los años 50, los problemas de una posición de frecuentista se hicieron obvios y eso cambió completamente la actitud hacia la probabilidad. Pero el concepto de propensión de Popper (1957) no es más que una elegante redacción del problema sin solución. Según esto, la probabilidad es una propiedad física de un objeto o situación para producir eventos - una propensión. Para una discusión de los fundamentos de la probabilidad, ver también Batanero, Henry y Parszysz (2005), o Batanero, Chernoff, Engel, Lee y Sánchez (2016).
} 
importante aunque sólo cubre un caso especial de probabilidad, es decir, cuando finitamente muchos resultados posibles tienen la misma probabilidad.

Con algunas restricciones, las dos teorías de la probabilidad (FQT, SJT) pueden ser reconciliadas por un cuerpo único de matemáticas; sin embargo, las afirmaciones seguirían teniendo connotaciones diferentes y sirviendo a diferentes intuiciones. Esto se hace evidente en la inferencia estadística, que llevó a la controversia bayesiana en los fundamentos. La visión de la APT está fuertemente interconectada con los aspectos psicológicos de la probabilidad, ya que existe una fuerte tendencia - según Lecoutre (1992) - a juzgar los diversos casos posibles como igualmente probables independientemente del contexto y "contra todo pronóstico" (es decir, incluso si la equiprobabilidad se pierde en una situación). La terminología de FQT, SJT y APT se remonta a Borovcnik y Kapadia (2014) y puede ser útil para diferenciar entre las escuelas y las connotaciones de probabilidad.

A continuación, nos centramos en la relevancia de las intuiciones y en cómo pueden ser utilizadas para diseñar intervenciones de enseñanza.

- Fischbein $(1975,1987)$ considera que la adquisición del concepto individual está determinada por una interacción entre las intuiciones primarias en bruto y las aportaciones teóricas de las tareas, reglas y conceptos de aprendizaje que tienen un aspecto más formal e inducen - además de aprender reglas matemáticas intuiciones secundarias, a menudo impregnadas de imágenes o situaciones paradigmáticas. Si las intuiciones secundarias son lo suficientemente fuertes, superan las intuiciones primarias de manera efectiva; si no, si los conceptos teóricos permanecen aislados para que el aprendizaje no tenga un efecto sustentador y los individuos regresen a sus intuiciones primarias en caso de que necesiten un juicio o ayuda.

- En Borovcnik (2011) se pueden encontrar argumentos que explican la dificultad de adquisición de un concepto operativo (Piaget \& Inhelder 1951) para la probabilidad. Una es la falta de retroalimentación para el uso de una estrategia adecuada en situaciones de probabilidad: se puede aplicar una estrategia sin sentido para encontrar, por ejemplo, los números en la lotería y ganar, o se puede tener un modelo adecuado para un fenómeno aleatorio $\mathrm{y}$, sin embargo, la decisión resulta errónea (Borovcnik \& Bentz 1990, 2003).

- Otra explicación es la discusión actual sobre las ideas fundamentales de estocástica, el pensamiento estocástico, el razonamiento estadístico, etc., que 
apunta en la dirección de una forma de pensamiento en lugar de simplemente aplicar una teoría matemática (rutinariamente). Hasta ahora, no hay un consenso sobre una lista razonable de ideas fundamentales de probabilidad que incluya una descripción adecuada de sus características relevantes; el intento inicial de Heitele (1975) se parece más a encabezados relacionados con el contenido de un libro de texto convencional que un catálogo de ideas fundamentales. Tal vez sea posible formular ideas fundamentales en torno a la noción de información, el tipo específico de información probabilística y las operaciones sobre la información como revisar (probabilidades condicionales), aumentar el nivel de información (aumentar el tamaño de la muestra), o transferir información de las muestras a las poblaciones si la información se obtiene al azar (inferencia estadística).

- Se añade la relevancia de las intuiciones frente a las operaciones, que puede verse en numerosos estudios de investigación y un intento convincente de operacionalizar el concepto de alfabetización estadística (Gal 2002), pero -hasta la fecha - rara vez existe un documento sobre la alfabetización probabilística (con la excepción de Borovcnik 2006, o Batanero \& Borovcnik 2016). Describir el constructo de la alfabetización probabilística es un tema abierto (ver Batanero, Chernoff, Engel, Lee, \& Sánchez 2016) aunque hay intentos rudimentarios por parte de Gal (2005; basado en una transferencia de la alfabetización estadística de Gal 2002).

Por último, la abundancia de paradojas (Székely 1986) y el carácter teórico de la probabilidad, según el cual el concepto se desmorona y ya no puede entenderse si se centra en un solo aspecto - como defiende Steinbring (1991) - son otros argumentos que podrían presentarse para seguir el enfoque intuitivo de Fischbein (1975). Spiegelhalter (2014a) destaca el carácter sofisticado de la probabilidad; Spiegelhalter (2014b) se refiere a un carácter metafórico de la probabilidad, que va mucho más allá de la materialización habitual por frecuencias relativas, que son el foco central de los actuales esfuerzos educativos en materia de probabilidad, de modo que incluso se puede afirmar que - en la práctica didáctica actual - la probabilidad se reduce erróneamente a su aspecto de frecuencias relativas, que se materializan por simulación. 


\subsection{Diseño De Los Applets Dinámicos Para Apoyar La Adquisición De Conceptos}

Un enfoque más directo más allá de la exposición matemática de los teoremas es un requisito básico de la educación estadística no sólo para los estudiantes de estudios diferentes a las matemáticas. Además, el enfoque dentro de las matemáticas se centra en gran medida en la derivación de las conexiones matemáticas y su prueba lógica en relación con los axiomas y los criterios de optimización.

Por ejemplo, el teorema del límite central no está abierto a una prueba completa ni siquiera para los estudiantes de matemáticas; como las afirmaciones de estos teoremas dominan la interpretación de la probabilidad y se centran en el propósito de la probabilidad, es tan importante encontrar un camino de aprendizaje directo a las afirmaciones básicas que desarrolla una comprensión intuitiva sin desarollar los detalles matemáticos. ${ }^{7}$ Aclarar estas cuestiones mejora la comprensión del teorema del límite central y la importancia resultante de la distribución normal (incluso para las estadísticas no paramétricas). Presentamos visualizaciones dinámicas de inspiración didáctica, que hemos implementado en nuestros cursos introductorios (para estudiantes de matemáticas y otros estudios) con el fin de apoyar la comprensión de los conceptos más complejos. Nuestros principios en el diseño de los applets se reflejan en las siguientes ideas:

- Un concepto abstracto de probabilidad puede ilustrarse por su impacto en las frecuencias relativas en experimentos repetidos, independientemente de la connotación de probabilidad.

- El significado de los parámetros de los modelos de probabilidad puede mejorarse mostrando el efecto de los cambios sistemáticos de estos parámetros.

- Los teoremas centrales (teoremas de límite) son facilitados por simulación; sin embargo, en lugar de "mostrar" un comportamiento límite, que no puede ser representado en forma material sin perder su verdadero significado.

\footnotetext{
${ }^{7}$ Existe consenso en la comunidad educativa estadística en que la enseñanza de la probabilidad y la estadística deben encontrar vías de aprendizaje que permitan una percepción directa de las afirmaciones y la relevancia de teoremas centrales como la ley de los grandes números y el teorema del límite central. Estos teoremas están - matemáticamente - más allá del nivel de la escuela secundaria. Sin embargo, los teoremas dominan la interpretación de la probabilidad (como "límite" de frecuencias relativas) y explican la importancia de la distribución normal. Especialmente el uso de la distribución normal para la distribución muestral de la media debe nombrarse aquí. Las simplificaciones de las afirmaciones de estos teoremas pueden verse en ejemplos de Borovcnik (2015a) o Batanero y Borovcnik (2016). Estos ejemplos corroboran que es posible enseñar el significado de estos teoremas sin desarrollar los detalles matemáticos. El método habitual de enseñanza es simular las muestras y analizar los datos simulados para mostrar las propiedades relevantes. Este análisis de datos se apoya en pantallas gráficas que facilitan el acceso a las propiedades.
} 
- Nos enfocamos en establecer un patrón estable para un número fijo de experimentos y luego comparamos este patrón con otro escenario con un mayor número de experimentos. Tal comparación puede inspirar un experimento de pensamiento que finalmente mejore la compresión del teorema para los estudiantes.

Todos los applets comparten la exploración de un cambio dinámico de la situación inicial. Como en un video, se observan las diferentes etapas de surgimiento de una relación entre los conceptos investigados: Identificar los conceptos clave; Aclarar los conceptos; Vincular a interpretaciones; Vincular a inferencias; Volver a plantear problemas con ellos; Superar el problema mediante applets adecuados.

Una biblioteca con applets está disponible del autor. Estos applets han sido probados y evaluados en cursos universitarios durante varios años y revisados por la retroalimentación de los estudiantes. Por su carácter dinámico, pueden revelar - más allá y paralelamente a las consideraciones matemáticas - propiedades clave de la probabilidad.

\section{Conclusiones Y Perspectivas}

Es esencial respaldar las intuiciones con experimentos adecuados. En lugar de investigar el comportamiento de los experimentos cuando el tamaño de la muestra aumenta hasta el infinito, lo cual no es posible, investigamos un patrón estable a un tamaño de muestra fijo. Luego lo comparamos con un tamaño de muestra mayor (preferiblemente cuatro veces mayor). Comparar el patrón permite comprender el tipo de ley probabilística que se queda atrás. Esta estrategia permite establecer intuiciones sobre la tendencia y la variabilidad.

Hay experimentos de este tipo en Batanero y Borovcnik (2016) y también en Borovcnik (2015a). Se refieren a probabilidades condicionales, a la aditividad de los valores esperados, a la aditividad de la dispersión en el caso de muestras independientes, a comparaciones informales de una distribución de probabilidad que representa la población y a los resultados de una muestra única (lo que conduce a una inferencia informal) y a otros aspectos de la probabilidad introductoria con una transición sin fisuras de las estadísticas de probabilidad a las inferenciales. Para las probabilidades condicionales existe también una versión multiplicativa de la fórmula bayesiana basada en las llamadas (cociente de) probabilidades, que proporcionan una representación mucho más adecuada de la información probabilística para las probabilidades condicionales. 
Integrar animaciones, analogías y simulaciones es más que motivador; puede aclarar mucho más los conceptos y cambiar las intuiciones de una manera más sustentable de lo que lo haría un enfoque matemático unilateral. En coherencia con nuestro enfoque en el valor de las intuiciones y la interacción dinámica entre las intuiciones primarias e intuiciones primarias y las tareas, imágenes o desafíos matemáticos, tratamos de diseñar intervenciones didácticas que desarrollen intuiciones secundarias a partir de los encuentros "matemáticos". Estas intuiciones secundarias se sitúan todavía en el nivel intuitivo, de modo que apoyen una comprensión "ganzheitlich" de los conceptos sin necesidad de todo detalle matemático. Esto sería aún más importante para la probabilidad que para cualquier otra parte de las matemáticas, de modo que los alumnos puedan integrar con éxito los conceptos de probabilidad y riesgo no sólo en su propio sistema cognitivo sino también en su mundo real, que está lleno de incertidumbre y riesgo.

Aquí nos hemos centrado en los aspectos teóricos de las ideas clave de la probabilidad y en cómo proporcionar intuiciones e imágenes que sean sostenibles. Dentro del proyecto "Conceptos fundamentales y sus propriedades clave en probabilidad" hay otros artículos en preparación sobre el desarrollo de tales ideas mediante intervenciones de enseñanza. Uno se centrará en contextos, analogías y tareas, que exponen el propósito de los conceptos clave de probabilidad; el otro revelará conceptos clave que están relacionados con los dos enfoques principales hacia la probabilidad y la inferencia estadística: el enfoque clásico y el enfoque bayesiano, que están conectados con las dos interpretaciones principales de la probabilidad: la visión del frecuentista (FQT) y la visión subjetivista (SJT) (Borovcnik \& Kapadia 2014). Resulta útil destacar ambos enfoques (FQT y SJT), ya que una reducción a una interpretación de la probabilidad generalmente conduce a enormes dificultades para entender la probabilidad, como lo han demostrado de manera convincente Carranza y Kuzniak (2008) en sus estudios. Esta perspectiva plural de la probabilidad (Chernoff \& Sriraman 2014) debería contrarrestar las desventajas de la tendencia de la educación estadística de los últimos veinte años a reducir la probabilidad a su aspecto frecuentista (FQT).

\section{Referencias}

Batanero, C. (2016). Understanding randomness: Challenges for research and teaching. In K. Krainer and N. Vondrová (Eds.), Proceedings of the Ninth Congress of the European Society for Research in Mathematics Education (pp. 34-49). Prague: ERME.

Batanero, C. \& Borovcnik, M. (2016). Statistics and probability in high school. Rotterdam: 
Sense.

Batanero, C., Chernoff, E., Engel, J. Lee, H., \& Sánchez, E. (2016). Research on teaching and learning probability. ICME-13 Topical Surveys. Cham, Switzerland: Springer.

Batanero, C., Henry, M., \& Parzysz, B. (2005). The nature of chance and probability. In: A. G. Jones (Ed.), Exploring probability in school (pp. 15-37). New York: Springer.

Bennett, D. J. (1999). Randomness. Cambridge, MA: Harvard University Press.

Borovcnik, M. (1992). Stochastik im Wechselspiel von Intuitionen und Mathematik (Stochastics in the interplay between intuitions and mathematics). Mannheim: Bibliographisches Institut.

Borovcnik, M. (2006). Probabilistic and statistical thinking. In M. Bosch (Ed.), Proceedings of the Fourth Congress of the European Society for Research in Mathematics Education (pp. 484-506). Barcelona: European Society for Research in Mathematics Education.

Borovcnik, M. (2011). Strengthening the role of probability within statistics curricula. In C. Batanero, G. Burrill, \& C. Reading (Eds.), Teaching statistics in school mathematicschallenges for teaching and teacher education. (pp. 71-83). New York: Springer.

Borovenik, M. (2012). Multiple perspectives on the concept of conditional probability. Avances de Investigación en Didactica de la Matemática, 1(2), 5-27.

Borovcnik, M. (2015a). Central theorems of probability theory and their impact on probabilistic intuitions. In J. M. Contreras, et al. (Eds.), Didáctica de la Estadística, Probabilidad y Combinatoria, 2 (pp. 15-35). Granada, Universidad de Granada.

Borovcnik, M. (2015b). Risk and decision making: The "logic" of probability. The Mathematics Enthusiast, 12(1, 2 \& 3), 113-139.

Borovcnik, M., \& Bentz, H. J. (1990, 2003). Intuitive Vorstellungen von Wahrscheinlichkeitskonzepten: Fragebögen und Tiefeninterviews (Intuitive conceptions of probability: Questionnaire and in-depth interviews). Technical Reports. Klagenfurt University.

Borovcnik, M., Kapadia, R. (2011). Modelling in probability and statistics - Key ideas and innovative examples. In J. Maaß, \& J. O’Donoghue (Eds.), Real-World Problems for Secondary School Students-Case Studies (pp. 1-44). Rotterdam: Sense Publishers.

Borovcnik, M., \& Kapadia, R. (2014). A historical and philosophical perspective on probability. In E. J. Chernoff \& B. Sriraman (Eds.), Probabilistic thinking: presenting plural perspectives (pp. 7-34). New York: Springer.

Borovcnik, M. \& Kapadia, R. (2018). Reasoning with risk: Teaching probability and risk as twin concepts. In C. Batanero \& E. J. Chernoff (Eds.), Teaching and Learning Stochastics - Advances in Probability Education Research (pp. 3-22). ICME 13 Topic Series. Cham, Switzerland: Springer International Publishing. 
Carranza, P. \& Kuzniak, A. (2008). Duality of probability and statistics teaching in French education. In C. Batanero, G. Burrill, C. Reading, \& A. Rossman (Eds.), Joint ICMI/IASE Study: Teaching Statistics in School Mathematics. Challenges for Teaching and Teacher Education. Monterrey: ICMI and IASE.

Chernoff, E. J. (2013). Probabilistic relativism: A multivalentological investigation of normatively incorrect relative likelihood comparisons. Philosophy of Mathematics Education Journal, 27, $1-30$.

Chernoff, E. \& Sriraman, B. (Eds.) (2014). Probabilistic thinking: presenting plural perspectives. Advances in Mathematics Education (Vol. 7). New York: Springer.

de Finetti, B. (1937/1992). La prévision: ses lois logiques, ses sources subjectives. Annales Institut Henri Poincaré, 7, 1-68 (1937); English translation: Foresight: Its logical laws, its subjective sources. In S. Kotz, \& N. L. Johnson (Eds.), Breakthroughs in statistics. (pp. 134-174). New York, Berlin: Springer.

de Finetti, B. (1974). Theory of probability. New York: Wiley (Translated by A. Machi \& A. Smith).

David, F. N. (1962). Games, gods and gambling. London: Griffin.

Dubben, H.-H., \& Beck-Bornholdt, H.-P. (2010). Mit an Sicherheit grenzender Wahrscheinlichkeit. Logisches Denken und Zufall. Reinbek: Rowohlt.

Dürr, D., Goldstein, S., Tumulka, R., \& Zanghi, N. (2004). Bohmian mechanics and quantum field theory. In: Physical Review Letters, 93(9).

Fischbein, E. (1975). The intuitive sources of probabilistic thinking in children. Dordrecht: Reidel.

Fischbein, E. (1987). Intuition in science and mathematics. Dordrecht: Reidel,

Gal, I. (2002). Adults' statistical literacy: Meanings, components, responsibilities (with discussion). International Statistical Review, 70(1), 1-51.

Gal, I. (2005). Towards "probability literacy" for all citizens: Building blocks and instructional dilemmas. In G.A. Jones (Ed.), Exploring probability in school (pp. 39-63). Dordrecht: Kluwer.

Gigerenzer, G. (2002). Calculated risks: How to know when numbers deceive you. New York: Simon \& Schuster.

Hacking, I. (1975). The emergence of probability. Cambridge: Cambridge University Press.

Heitele, D. (1975). An epistemological view on fundamental stochastic ideas. Educational Studies in Mathematics, 6, 187-205. 
Jones, G. A., Langrall, C.W., \& Mooney, E. S. (2007). Research in probability: Responding to classroom realities. In F. K. Lester (Ed.), Second handbook of research on mathematics teaching and learning, Vol. 2 (pp. 909-956). Greenwich, CT: Information Age Publishing.

Kahneman, D. \& Tversky, A. (1972). Subjective probability. A judgment of representativeness. Cognitive Psychology 3(3), 430-454.

Kahneman, D. \& Tversky, A. (1973). Availability: A heuristic for judging frequency and probability. Cognitive Psychology, 5(2), 207-232

Kahneman, D. \& Tversky, A. (1979). Prospect theory: An analysis of decision under risk. Econometrica, 47(2), 263-292.

Kahneman, D., Slovic, P., \& Tversky, A. (1982). Judgment under uncertainty: Heuristics and biases. Cambridge: Cambridge University Press.

Kapadia, R., Borovcnik, M. (Eds.) (1991). Chance encounters. Probability in education. Dordrecht: Kluwer.

Kolmogorov, A. N. (1933/1956). Grundbegriffe der Wahrscheinlichkeitsrechnung. Berlin; English translation: Foundations of the theory of probability. New York: Chelsea.

Konold, C. (1989). Informal conceptions of probability. Cognition and Instruction, 6(1), 59-98.

Laplace, P. S. de (1814/1995). Théorie analytique des probabilités, 2nd ed. Paris: Courcier; 1st ed. 1812; reprinted 1995. Paris: Jacques Gabay.

Lecoutre, M. P. (1992). Cognitive models and problem spaces in "purely random" situations. Educational Studies in Mathematics, 23(6), 557-568.

Mises, R. v. (1919). Grundlagen der Wahrscheinlichkeitsrechnung. Mathematische Zeitschrift, 5, 52-99.

Piaget, J. \& Inhelder, B. (1951). La genèse de l'idée de hasard chez l'enfant (The origin of the idea of chance in children). Paris: Presses Universitaires de France.

Simons, P. R. (1984). Instructing with analogies. Journal of Educational Psychology, 76(3), $513-527$.

Spiegelhalter, D. (2014a). Probabilistic thinking. In: E. J. Chernoff \& B. Sriraman (Eds.), Probabilistic thinking: presenting plural perspectives (Back cover). New York: Springer.

Spiegelhalter, D. (2014b, April). What can education learn from real-world communication of risk and uncertainty? Eight British Congress on Mathematical Education, Nottingham.

Steinbring, H. (1991). The theoretical nature of probability in the classroom. In: R. Kapadia \& M. Borovcnik (Eds.). Chance encounters (pp. 135-167). Dordrecht: Kluwer.

Styer, D. F. (2000). The strange world of quantum mechanics. Cambridge: Cambridge University Press. 
Székely, G.J. (1986). Paradoxes in probability and mathematical statistics. Dordrecht: Reidel.

Tversky, A. \& Kahneman, D. (1974). Judgment under uncertainty: Heuristics and biases. Science, 185, 1124- 1130.

von Plato, J. (1994). Creating modern probability: its mathematics, physics and philosophy in historical perspective. Cambridge: Cambridge University Press.

Recebido em: 10/09/2019

Aceito em: 17/09/2019 